\title{
Preservation of Postharvest Quality of Leafy Amaranth (Amaranthus spp.) Vegetables Using Evaporative Cooling
}

\author{
Jane Ambuko, ${ }^{1}$ F. Wanjiru, ${ }^{1}$ G. N. Chemining'wa, ${ }^{1}$ W. O. Owino, ${ }^{2}$ and Eliakim Mwachoni1 \\ ${ }^{1}$ Department of Plant Science \& Crop Protection, University of Nairobi, P.O. Box 29053, Nairobi, Kenya \\ ${ }^{2}$ Department of Food Science \& Technology, Jomo Kenyatta University of Agriculture \& Technology, Nairobi, Kenya \\ Correspondence should be addressed to Jane Ambuko; ambuko@yahoo.com
}

Received 7 September 2017; Accepted 15 November 2017; Published 12 December 2017

Academic Editor: María B. Pérez-Gago

Copyright (c) 2017 Jane Ambuko et al. This is an open access article distributed under the Creative Commons Attribution License, which permits unrestricted use, distribution, and reproduction in any medium, provided the original work is properly cited.

\begin{abstract}
Leafy vegetables are very highly perishable and must be utilized immediately after harvest. Their fast deterioration is attributed to various biological and environmental factors with temperature playing a central role. Evaporative cooling is a low-cost temporary storage technology that offers smallholder vegetable farmers an alternative to expensive cold rooms. The present study sought to determine the effectiveness of evaporative cooling using zero energy brick cooler (ZEBC) and evaporative charcoal cooler (ECC), to preserve the postharvest quality of leafy amaranth vegetables. Freshly harvested vegetables were separated into bundles weighing 300 grams and stored under ZEBC, ECC, and ambient room conditions (control). Real time changes in temperature and relative humidity $(\mathrm{RH})$ as well as changes in quality attributes (physiological weight loss (PWL), wilting index, hue angle, and vitamin $\mathrm{C}$ ) were determined during the storage period. The temperature difference between the ZEBC and ECC versus the ambient air ranged between 4 and $10^{\circ} \mathrm{C}$. Significantly higher RH (80-100\%) was recorded in both evaporative cooling chambers. At the end of storage, higher PWL (47.6\%) was recorded at ambient room conditions compared to 10.5 and $6.7 \%$ under ZEBC and ECC, respectively. A rapid decline in vitamin C (51\%) was reported in vegetables stored at ambient room conditions. Overall, there was better vegetable quality preservation under ECC and ZEBC.
\end{abstract}

\section{Introduction}

Fruits and vegetables are a rich source of dietary fiber and micronutrients such as vitamin $\mathrm{C}$, thiamin, riboflavin, B-6, niacin, folate, $\mathrm{A}$, and $\mathrm{E}$. Phytochemicals in fruits and vegetables, such as polyphenolics, carotenoids, and glucosinolates, may also have nutritional value [1]. African leafy vegetables such as leafy amaranth have received great attention as a cheap and readily available source of micronutrients for lowincome rural communities [2]. The interest in vegetables (and fruits) has increased over the last decade in part due to increased consumer awareness about their health benefits. Leafy vegetables deteriorate very rapidly after harvest and therefore require proper postharvest handling to preserve the quality at harvest. Lack of knowledge on appropriate quality preservation practices and technologies can result in high qualitative and quantitative losses in vegetables [3]. High postharvest losses (upwards of 50\%) in leafy vegetable are attributed to various biological and environmental factors.
Loss of quality and deterioration in harvested leafy green vegetables is manifested through yellowing as a result of loss of chlorophyll; wilting and loss of textural properties; and decay from pathological breakdown, among others. Transpirational water loss is one of the physiological processes that results in deterioration of leafy vegetables. It results in loss of freshness as evidenced by wilting, shriveling, and loss of firmness, crispness, and succulence, which all are components of freshness. If leafy vegetables lose more than $3 \%$ of the original fresh weight, they are rendered unsalable [4]. In addition to deterioration and loss of physical component of quality, vitamin $C$ which is a major micronutrient in vegetables is known to decline rapidly after harvest. Loss of vitamin C is often used as an indicator of quality deterioration during postharvest handling including transportation, storage, and processing because it is highly susceptible to chemical and enzymatic oxidation and is highly water soluble [1].

The rate of deterioration of leafy vegetables is determined by the storage environment where temperature and relative 
humidity are key components. Most perishable horticultural commodities (chilling insensitive commodities) have an optimal shelf-life at temperatures of approximately $0^{\circ} \mathrm{C}$. The rate of deterioration of perishables however increases two- to threefold with every $10^{\circ} \mathrm{C}$ increase in temperature therefore for most perishable commodities there is a loss of storage potential as handling temperatures increase [5]. Consequently maintaining low (safe) temperature during handling of the commodity right from harvest to retail stage (cold chain) is critical for the preservation of the quality of perishable commodities and reducing postharvest losses.

In technologically advanced developed countries, cold storage using conventional (mechanical) refrigeration is an integral part of postharvest handling of perishable horticultural commodities. However, majority of the smallholder farmers in developing countries do not have access to conventional cold rooms. This is partly due to the high cost of purchase and maintenance of such facilities. Nonuse of the conventional cold rooms is also attributed to lack of connection to the national grid in most rural areas where horticultural farming is practiced. As a solution to the expensive and inaccessible conventional cold rooms, other low-cost cold storage options have been developed. Among these technologies are those that are based on evaporative cooling. Evaporative cooling is premised on the evaporative heat exchange which takes advantage of the principles of the latent heat of evaporation where tremendous heat is exchanged when water evaporates. Water is in constant state transition from solid to liquid to vapor. To move from one state to the other, water takes or loses heat. For example change of water from liquid to vapor requires $2260 \mathrm{~kJ} / \mathrm{kg}$ of water $=$ latent heat of vaporization. This heat transfer through the evaporation of water is the basis of evaporative cooling technologies.

Evaporative cooling technologies take many forms but basically comprise a porous wall made of a medium that can hold water and through which air can easily flow. The medium could be charcoal, sand, clay, or any other material that is porous enough to hold water. This medium is kept wet and as dry/hot air flows over it, the water therein evaporates resulting in a cooling and humidifying effect [6]. The measure of effectiveness is based on how close the air temperature leaving the evaporative cooler approaches the outdoor wet bulb temperatures [6].

Previous studies have shown that evaporative cooling can be used to attain significantly lower than ambient temperatures and higher than ambient relative humidity. Studies in India showed that a high relative humidity (85-90\%) in a zero energy cool chamber (ZECC) compared to $21-94 \%$ in the case of the shed and field which showed great fluctuation [7]. Similarly, relatively low temperatures were attained in ZECC with less fluctuation in maximum and minimum temperatures compared to shed and field conditions. Under ZECC storage, the shelf-life of fruits and vegetables increased with lower physiological weight loss recorded compared to room temperature [7]. A $10-15^{\circ} \mathrm{C}$ temperature difference between the inside of an evaporative cool chamber and the outside was reported by [8]. In the same chamber, the $\mathrm{RH}$ inside was $30-40 \%$ higher than the outside. Under these conditions, wilting and loss of freshness were significantly slowed thereby keeping the fruits and vegetables fresh for up to 3-5 days more inside the chamber than outside.

The main advantage of evaporative cooling technologies is the fact that they do not require mechanical or electrical energy input to operate and are therefore appropriate for smallholder farmers in rural areas without electricity. Additionally, the chambers can be constructed from locally available materials using unskilled labor thereby making the cost affordable for the resource-poor smallholder farmers. The evaporative cooling units are considerably cheap. A small unit with a $200 \mathrm{Kg}$ storage capacity can cost between USD 200 and $300[9,10]$. Larger units (1 MT capacity) can be built at an estimated cost of USD 1,000 [9].

Despite the advantages and potential to preserve postharvest quality of fruits and vegetables, the adoption of evaporative cooling technologies among smallholder farmers is still low. Promotion of these technologies requires evidence of their effectiveness to attain conducive storage conditions and consequently preserve quality of perishable commodities. The objective of this study was therefore to evaluate the effectiveness of evaporative cooling using ZEBC and ECC to preserve the postharvest leafy amaranth.

\section{Materials and Methods}

2.1. Experimental Site and Materials. The study was carried out at the field station of the University of Nairobi, College of Agriculture and Veterinary Sciences, Nairobi, Kenya, between January and May 2015. Leafy amaranth vegetables were harvested early in the morning and immediately transported to the packing house. The vegetables were then separated into uniform bundles of approximately equal weight (300 g).

2.2. Storage Chambers. A zero energy brick cooler (ZEBC) was built from locally available materials including earthen bricks, river bed sand, sisal sacks, sisal waste, fiber board, water tank, and water drip lines. The bricks were arranged to make a double wall measuring $220 \times 200 \mathrm{~cm}$ and $60 \mathrm{~cm}$ high. Wet river bed sand was sandwiched between the two walls. The water in the sand was replenished through a drip line connected to a water reservoir. The top cover of the ZEBC was made from fiberboard and sisal gunny bags stuffed with sisal waste (a byproduct of sisal products processing). The evaporative charcoal cooler (ECC) was made up of a double wall made of chicken wire stuffed with charcoal. The charcoal was wetted using a drip line just like in the case of the ZEBC. The bundled vegetables were arranged in plastic crates and stored in either the ZEBC or ECC under ambient room conditions (control). In each of the three storage environments, data loggers (Xsense) were fitted to measure the changes in temperature and relative humidity during the storage period.

2.3. Data Collection. Three bundles of vegetables were sampled from each of the storage environments every 2 days for determination of changes in quality attributes including cumulative weight loss, color, wilting index, and vitamin 


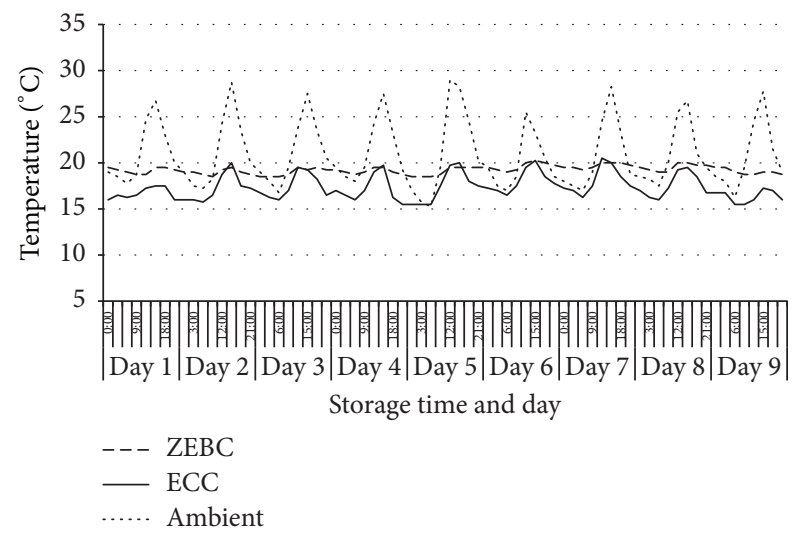

Figure 1: Changes in temperature in the zero energy brick cooler (ZEBC), evaporative charcoal cooler (ECC), and ambient room conditions.

C. Cumulative weight loss was determined using a digital weighing balance (Dymo, M5 model, China) and expressed as weight lost as percentage of the initial weight. Color was determined using a TES color meter (Model Tes 135, Taipei, Taiwan) which was calibrated with a white calibration card. $L^{*}, a^{*}$, and $b^{*}$ coordinates were determined and $a^{*}$ and $b^{*}$ values were converted to hue angle $\left(H^{\circ}\right)$ [11]. The extent of wilting was assessed on the basis of a 7-point hedonic scale, where 1 = extreme wilting, $2=$ very severe wilting, $3=$ severe wilting, $4=$ moderate wilting, $5=$ slight wilting, $6=$ very slight wilting, and $7=$ no wilting. Vitamin $C$ content was determined using the AOAC method [12]. The experimental design used was a completely randomized design (CRD) with three treatments and three replications. The data collected were subjected to analysis of variance (ANOVA) using a statistical package Genstat 15th edition and the means were separated by Least Significant Difference (LSD) at $p=0.05$.

\section{Results}

3.1. Temperature. The temperature fluctuated with the time of day with the highest day time temperature being $29^{\circ} \mathrm{C}$, while the lowest night temperature was $15.3^{\circ} \mathrm{C}$ (Figure 1). During this period, the temperature in the ZEBC ranged between 18.0 and $20.0^{\circ} \mathrm{C}$ with minimal fluctuation irrespective of the time of day. On the contrary, temperature in the ECC fluctuated between 15.5 and $20.5^{\circ} \mathrm{C}$ depending on the outside temperatures (Figure 1). Overall the temperature difference between the ambient room and the evaporative coolers (ZEBC and ECC) ranged between 1 and $10^{\circ} \mathrm{C}$ depending on the time of day.

3.2. Relative Humidity. Relative humidity (RH) at ambient room conditions fluctuated between 46 and 97\% (Figure 2). The lowest RH values (below 50\%) were recorded during the afternoons, while the highest values (>95\%) were recorded between 6 p.m. and 6 a.m. The RH values in the ECC ranged between 75 and $100 \%$ and fluctuated in a similar pattern as the $\mathrm{RH}$ at the ambient room conditions. On the contrary, the RH values in the ZEBC were stable at $100 \%$ regardless of the time of day (Figure 2). Overall the difference in $\mathrm{RH}$ values
TABLE 1: Physiological weight loss (\% of the initial weight) in amaranth vegetables (Amaranth spp.) stored in the zero energy brick cooler (ZEBC) and evaporative charcoal cooler (ECC) under ambient room conditions.

\begin{tabular}{lcccc}
\hline \multirow{2}{*}{ Storage option } & \multicolumn{4}{c}{ Days in storage } \\
& 0 & 2 & 5 & 8 \\
\hline ZEBC & 0 & $6.4^{\mathrm{a}}$ & $10.5^{\mathrm{b}}$ & $16.2^{\mathrm{a}}$ \\
ECC & 0 & $5.6^{\mathrm{a}}$ & $6.7^{\mathrm{b}}$ & $16.9^{\mathrm{a}}$ \\
Ambient room & 0 & $5.6^{\mathrm{a}}$ & $47.6^{\mathrm{a}}$ & - \\
\hline LSD & 1.56 & 12.12 & 9.20 \\
CV\% & 11.7 & 14.8 & 36.8 \\
Significance & & $n s$ & $*$ & $n s$ \\
\hline
\end{tabular}

Means within each column followed by different letters differ significantly at $p<0.05 .{ }^{*}$ Significantly different at $p<0.05$.

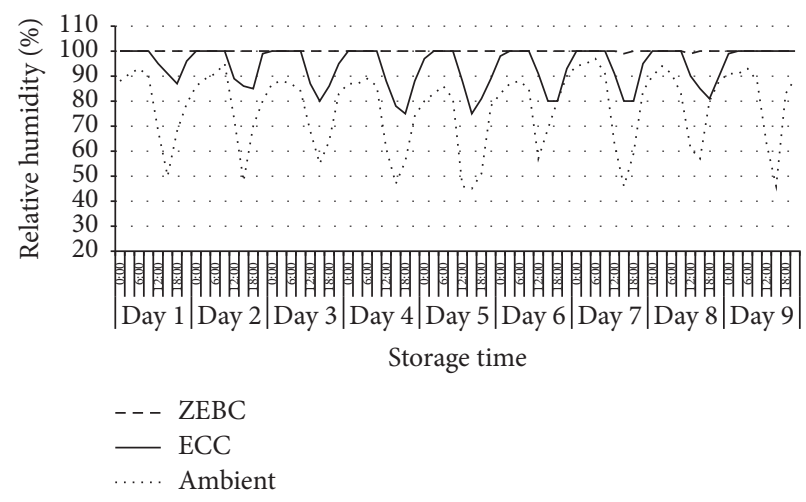

FIGURE 2: Changes in relative humidity in the zero energy brick cooler (ZEBC), evaporative charcoal cooler (ECC), and ambient room conditions.

between the ambient room conditions and the evaporative coolers ranged between 0 and $55 \%$ depending on the time of day.

3.3. Physiological Weight Loss (PWL) and Wilting Index. The vegetables lost some weight during storage regardless of the storage environment. By the 5th day of storage, the vegetables stored at ambient room conditions had lost $47.6 \%$ ( half) of the initial weight compared to 6.7 and $10.5 \%$ in the case of vegetables stored in the ECC and ZEBC, respectively (Table 1). At the end of the storage period (day 8), vegetables in the ECC and ZEBC had lost $16.9 \%$ and $16.2 \%$ of their initial weight, respectively. The trend in PWL was mirrored by the extent of wilting where the vegetables stored in the ZEBC and ECC wilted at a slower rate compared to those stored at ambient room conditions (Figure 3 ).

3.4. Hue Angle (Color Changes). The hue angle reduced gradually as the vegetables' color changed from the initial dark green color $\left(>130^{\circ}\right)$ to a lighter shade of green $\left(<120^{\circ}\right)$. There was no significant (statistical) difference in the hue angle of vegetables stored under the different storage conditions (Table 2). 
TABLE 2: Changes in hue angle of Amaranth spp. stored under zero energy brick cooler (ZEBC), evaporative charcoal cooler (ECC), and ambient room conditions.

\begin{tabular}{lcccc}
\hline \multirow{2}{*}{ Cooler } & \multicolumn{4}{c}{ Days in storage } \\
& 0 & 2 & 5 & 8 \\
\hline ZEBC & 138.54 & $129.22^{\mathrm{a}}$ & $118.92^{\mathrm{a}}$ & $115.05^{\mathrm{a}}$ \\
ECC & 138.54 & $125.94^{\mathrm{a}}$ & $124.89^{\mathrm{a}}$ & $121.27^{\mathrm{a}}$ \\
Ambient room & 138.54 & $126.86^{\mathrm{a}}$ & $129.30^{\mathrm{a}}$ & - \\
\hline Grand mean & & 127.34 & 124.37 & 85.44 \\
LSD & & 11.435 & 14.273 & 14.079 \\
CV\% & 4.0 & 5.1 & 7.3 \\
Significance & & $n s$ & $n s$ & $n s$ \\
\hline
\end{tabular}

Means within each column followed by different letters differ significantly at $p<0.05$.

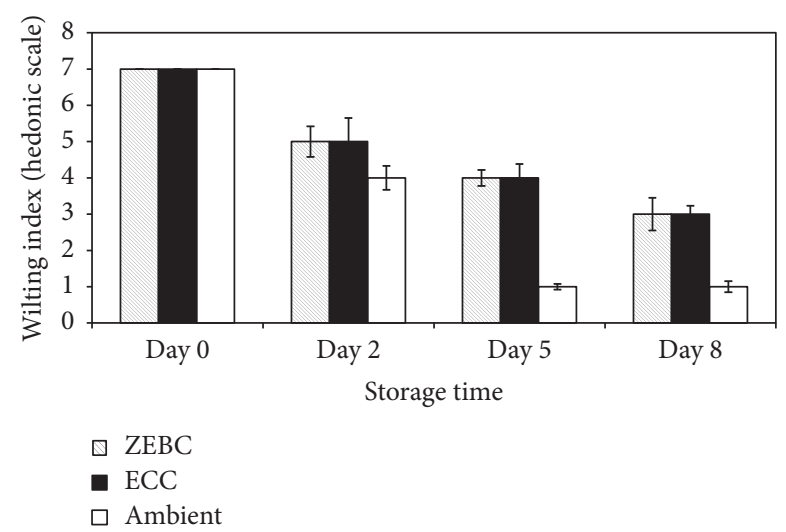

Figure 3: Wilting index of leafy Amaranth spp. stored under zero energy brick cooler (ZEBC), evaporative charcoal cooler (ECC), and ambient room conditions on a 7-point hedonic scale: $7=$ no wilting, $6=$ very slight wilting, $5=$ slight wilting, $4=$ moderate wilting, $3=$ severe wilting, $2=$ very severe wilting, and $1=$ extreme wilting.

3.5. Vitamin C. Vitamin C content in the vegetables reduced gradually during storage from the initial $19.5 \mathrm{mg} / 100 \mathrm{~g}$ to 10.8 and $11.3 \mathrm{mg} / 100 \mathrm{~g}$ at the end of storage (day 8) in the ZEBC and ECC, respectively (Table 3 ). The reduction was most dramatic in vegetables stored at ambient room conditions whereby at the end of storage (day 5) the vitamin $\mathrm{C}$ level had reduced to $8.4 \mathrm{mg} / 100 \mathrm{~g}$ which was $43 \%$ of the initial values.

\section{Discussion}

Fresh fruits and vegetables are a rich source of micronutrients and therefore their consumption especially among resourcepoor populations in developing countries is encouraged as a measure to mitigate against micronutrient deficiencies. However, preservation of the quality of these vegetables after harvest is critical to realization of this goal. Poor postharvest handling can result in loss of appreciable amounts of the nutrients present in the vegetables at harvest. It is therefore important to employ appropriate postharvest handling practices and technologies in order to preserve the quality of vegetables after harvest.
TABLE 3: Changes in vitamin C (mg/100 g fresh weight) of Amaranth spp. stored under zero energy brick cooler (ZEBC), evaporative charcoal cooler (ECC), and ambient room conditions.

\begin{tabular}{lcccc}
\hline \multirow{2}{*}{ Storage option } & \multicolumn{4}{c}{ Days in storage } \\
& 0 & 2 & 5 & 8 \\
\hline ZEBC & 19.5 & $15.7^{\mathrm{a}}$ & $12.6^{\mathrm{b}}$ & $10.8^{\mathrm{a}}$ \\
ECC & 19.5 & $14.3^{\mathrm{b}}$ & $13.4^{\mathrm{a}}$ & $11.3^{\mathrm{a}}$ \\
Ambient room & 19.5 & $10.3^{\mathrm{c}}$ & $8.4^{\mathrm{c}}$ & - \\
\hline LSD & & 0.84 & 0.59 & 1.79 \\
CV\% & 2.8 & 2.3 & 10.7 \\
Significance & & $* *$ & $* *$ & $n s$ \\
\hline
\end{tabular}

Means within each column followed by different letters differ significantly at $p<0.05$. $^{* *}$ Significantly different at $p<0.01$.

Maintaining a cold chain from harvest to the retail/consumption stage is critical in preserving quality and reduction of postharvest losses of perishable food commodities. A cold chain for perishable commodities is the uninterrupted handling of the commodity within a low temperature environment during the postharvest steps of the value chain including harvest, collection, packing, processing, storage, transport, and marketing until it reaches the final consumer [5]. Maintenance of cold chain is a challenge for majority of smallholder farmers in developing countries due to the associated costs and lack of connectivity to electrical energy. Evaporative cooling is a low-cost alternative to conventional refrigeration which is expensive and inaccessible to smallholder farmers in rural areas.

In the present study, the effectiveness of two evaporative cooling technologies, namely, zero energy brick cooler (ZEBC) and evaporative charcoal cooler (ECC), in preserving the postharvest quality of leafy amaranth vegetables was evaluated. The temperature difference between ambient room and the evaporative coolers ranged between 1 and $9.5^{\circ} \mathrm{C}$. The greatest difference in temperature was observed during the hottest time of the day (1200 to 1500 hours). During this time, the air temperature outside the chamber was at the highest point, while the relative humidity was the lowest point, hence a higher cooling capacity. Evaporation of water resulted in not only a cooling effect but also a rise in $\mathrm{RH}$ in the cooling chambers. The difference in RH between the chambers and ambient room ranged between 0 and 55\% depending on the time of day. The ZEBC maintained a stable high RH of $100 \%$.

These findings corroborate past reports on evaporative cooling technologies. A $10-15^{\circ} \mathrm{C}$ temperature difference and $\geq 90 \% \mathrm{RH}$ in a zero energy cool chamber with a similar design as the ZEBC were reported by [13]. In another study, a 5- $6^{\circ} \mathrm{C}$ temperature difference between the room conditions and earthen pot cool chamber with the $\mathrm{RH}$ ranging between 87 and $92 \%$ in the chamber was reported [14]. Similarly, studies in another version of evaporative cooler designed from clay reported a $10^{\circ} \mathrm{C}$ temperature reduction and increase in $\mathrm{RH}$ from 40.3 to $92 \%$ compared to ambient room conditions [15]. In the present study, under the modified storage environment, the quality of leafy amaranth vegetables was preserved for a longer time compared to those stored at ambient room conditions. The vegetables maintained a near 
farm-fresh state for an additional 2 days and a salable state for up to 5 days more than those stored at ambient room conditions. The lower temperature and high relative humidity slowed down the deteriorative processes including wilting and yellowing. In leafy vegetables, wilting is one of the major factors that contribute to not only loss of salable weight but also loss of aesthetic attributes which in turn affects salability of the vegetables. Vegetables contain $>90 \%$ water, hence very susceptible to water loss leading to wilting. Therefore, the vegetables remain fresh as long as they retain water. For most leafy vegetables, weight loss of up to $3 \%$ significantly affects their aesthetic value and salability. The rate of water loss is affected by temperature and $\mathrm{RH}$ which affect the vapor pressure difference (VPD) between the produce and its environment. In this regard, the higher the VPD, the higher the water loss [16]. The VPD can be reduced by lowering storage temperature and increasing RH. In the present study, the relatively lower temperature in the evaporative cooling chambers and high $\mathrm{RH}$ in the chambers contributed to a lower VPD and subsequently the reduced PWL and wilting and ultimately the longer shelf-life was observed.

By the 5th day of storage, vegetables that were stored at ambient room conditions had lost $45 \%$ of the initial vitamin $\mathrm{C}$ content. This rapid loss in vitamin $\mathrm{C}$ could be attributed to the relatively higher storage temperature and low $\mathrm{RH}$. Previous studies show that temperature and relative humidity are primary factors in preservation of vitamins in vegetables. Conditions that favor wilting were shown to result in a rapid loss of vitamin $\mathrm{C}$ in vegetables such as spinach, cabbage, snap beans, kales, and collards [17]. In broccoli, loss in vitamin $\mathrm{C}$ was shown to correlate positively with increase in storage temperature [18]. Although the color changes were not statistically different, the vegetables stored in the ECC and $\mathrm{ZEBC}$ maintained a greener color compared to the ones stored at ambient room conditions. This concurs with past studies in broccoli and kales where yellowing was shown to occur more rapidly as the storage temperature increased [18].

In conclusion, this study showed that storage of the perishable leafy amaranth vegetables in the ZEBC and ECC significantly slowed down wilting, yellowing, and loss of vitamin $C$. Overall, the vegetables stored in the $\mathrm{ZEBC}$ and ECC remained salable for an additional 3-5 days in comparison to the vegetables stored at ambient room conditions. For smallholder farmers in rural areas in developing countries such as Kenya, without alternative storage options, the additional days from evaporative cooling storage could buy the farmers more time to temporarily store the vegetables awaiting marketing. This will not only extend the marketing period for the vegetables but also allow the farmers to bulk the small quantities of vegetables often harvested by individual farmers. Ultimately this may contribute to goal of reducing postharvest losses in vegetables.

\section{Disclosure}

The funding source had no involvement in the study design; in the collection, analysis, and interpretation of data; in the writing of the report; and in the decision to submit the article for publication.

\section{Conflicts of Interest}

The authors declare that they have no conflicts of interest.

\section{Acknowledgments}

This work was supported by the German government through Horticultural Innovations and Learning for Improved Nutrition and Livelihood in East Africa (HORTINLEA) project, Grant Contracts: FKZ 031A248D and 031A248I.

\section{References}

[1] D. M. Barrett, "Maximizing the nutritional value of fruits \& vegetables," Food Technology, vol. 61, no. 4, pp. 40-45, 2007.

[2] E. O. Gogo, A. Opiyo, C. Ulrichs, and S. Huyskens-Keil, "Postharvest treatments of african leafy vegetables for food security in Kenya: a review," African Journal of Horticultural Science, vol. 9, pp. 32-40, 2016.

[3] J. N. Kinyuru, S. O. Konyole, G. M. Kenji et al., "Identification of traditional foods with public health potential for complementary feeding in western Kenya," Journal of Food Research (JFR), vol. 1, pp. 148-158, 2012.

[4] S. Ben-Yehoshua and V. Rodov, "Transpiration and water stress," in Postharvest Physiology and Pathology of Vegetables, J. A. Bartz and J. K. Brecht, Eds., pp. 111-159, Marcel Dekker, New York, NY, USA, 2nd edition, 2002.

[5] K. Lisa, "Use of cold chains for reducing food losses in developing countries," Postharvest Education Foundation (PEF) White Paper no. 13-03, 2013.

[6] A. Lal Basediya, D. V. K. Samuel, and V. Beera, "Evaporative cooling system for storage of fruits and vegetables - a review," Journal of Food Science and Technology, vol. 50, no. 3, pp. 429442, 2013.

[7] Roy S. K., Postharvest Technology of Vegetable Crops in India, Indian Horticulture, 1989.

[8] S. Dadhich, H. Dadhich, and R. Verma, "Comparative study on storage of fruits and vegetables in evaporative cool chamber and in ambient," International Journal of Food Engineering, vol. 4, no. $1,2008$.

[9] K. Lisa, Identification of Appropriate Postharvest Technologies for Improving Market Access and Incomes for Small Horticultural Farmers in Sub-Saharan Africa and South Asia, World Food Logistics Organization (WFLO) Grant Report, 2010.

[10] A. Jane, F. Wanjiru, E. Karithi et al., "Low-cost cold storage options for smallholder farmers to reduce postharvest losses in fruits and vegetables," in Proceedings of the 1st International Congress on Postharvest Loss Prevention, pp. 126-128, Rome, Italy, 2015.

[11] M. R. McLellan, L. R. Lind, and R. W. Kime, "Hue angle determination and statistical analysis for multiquadrant hunter L,a,b data," Journal of Food Quality, vol. 18, pp. 235-240, 1994.

[12] Association of Official Agricultural Chemists (AOAC), 967.22, Analytical method for Ascorbic Acid-L.in fruit juices and nectar.

[13] S. K. Roy and R. K. Pal, "A low cost zero energy cool chamber for short term storage of mango," Acta Horticulturae, vol. 291, pp. 519-524, 1991.

[14] A. M. Murugan, S. A. J. A. Ranjith, and S. Vidhya, "Evaluation of self-life and organoleptic aspects of fruits stored in a modified 
traditional earthen pot cool chamber," Indian Journal of Traditional Knowledge, vol. 10, no. 2, pp. 375-379, 2011.

[15] N. M. Chinenye, "Development of clay evaporative cooler for fruits and vegetables preservation," Agricultural Engineering International: CIGR Journal, vol. 13, no. 1, 2011.

[16] D. Holcroft, "Water relations in harvested fresh produce," The Postharvest Education Foundation (PEF) White Paper no. 1501, 2015.

[17] B. D. Ezell and M. S. Wilcox, "Vegetable vitamins, loss of vitamin $\mathrm{C}$ in fresh vegetables as related to wilting and temperature," Journal of Agricultural and Food Chemistry, vol. 7, no. 7, pp. 507509, 1959.

[18] M. Cantwell, UCCE Vegetable Pest Management and Postharvest Issues, Santa Maria, June, 2010. 

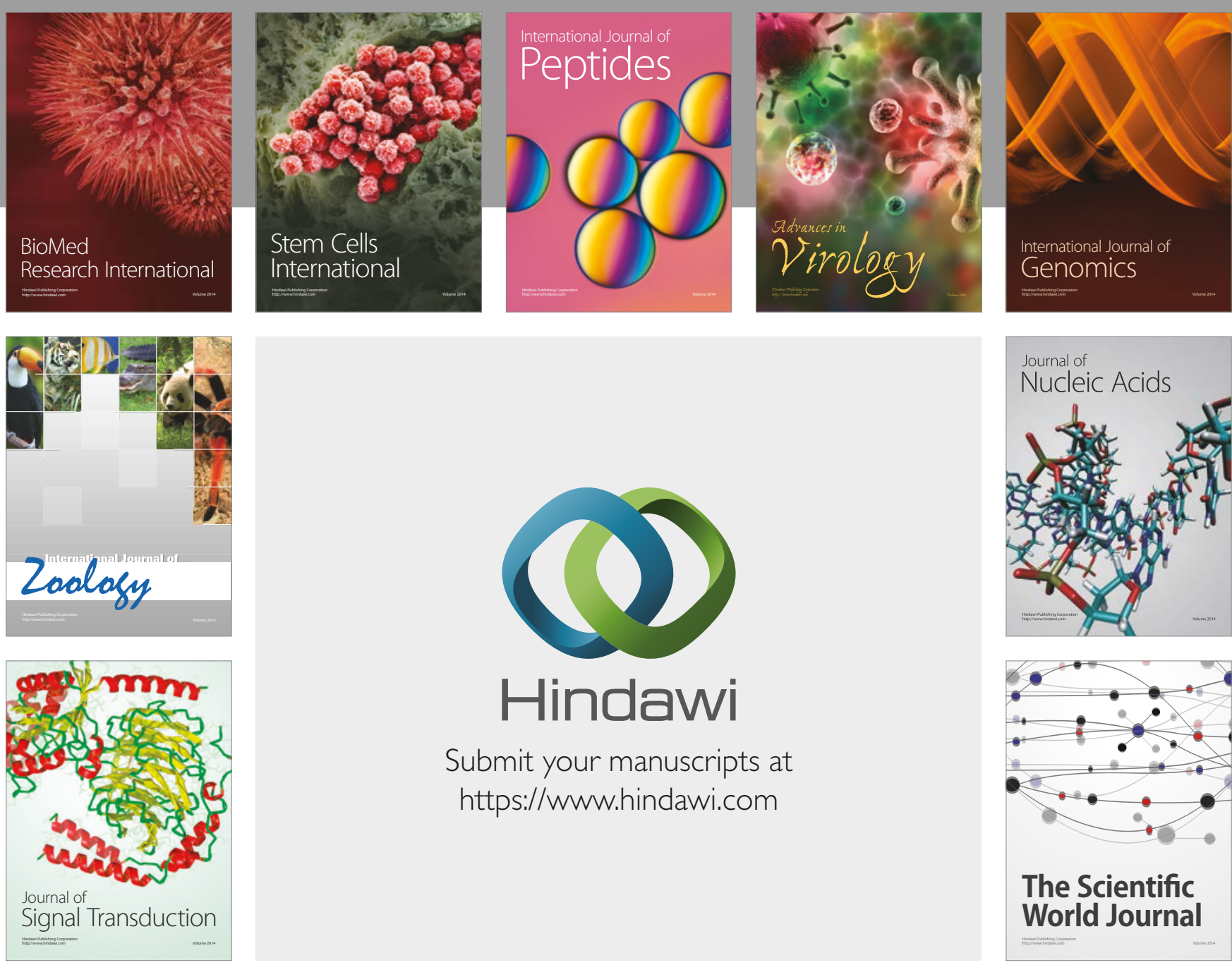

Submit your manuscripts at

https://www.hindawi.com
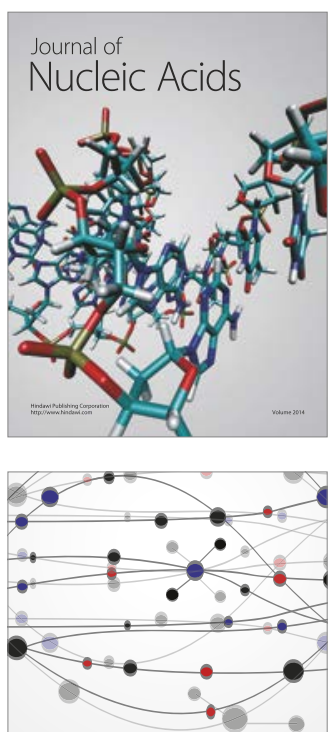

The Scientific World Journal

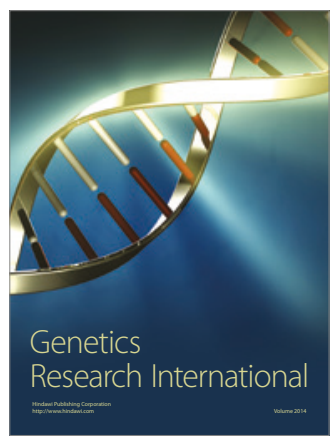

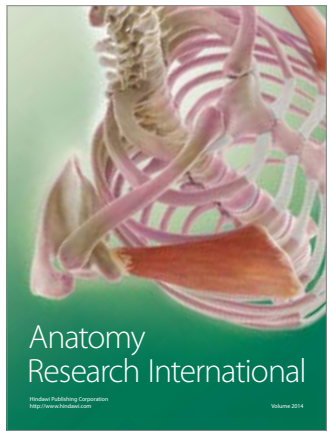

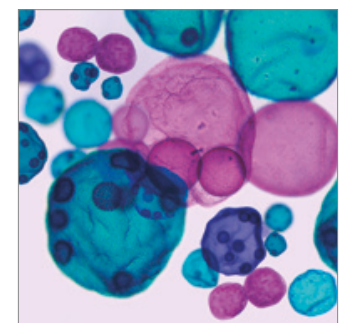

International Journal of Microbiology
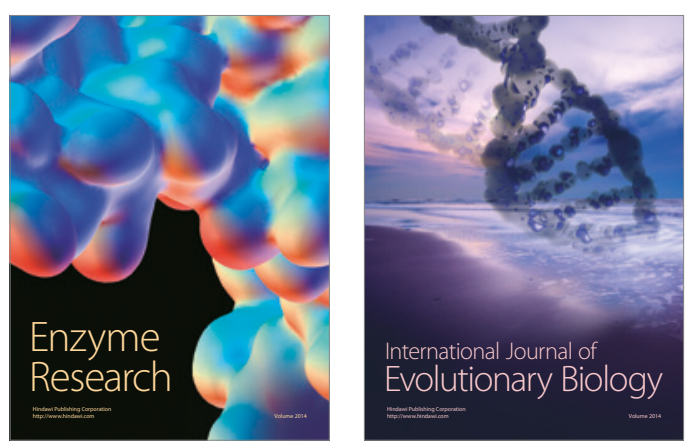
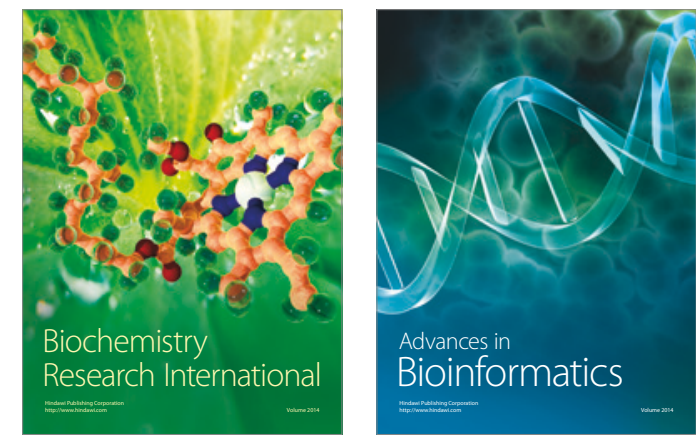

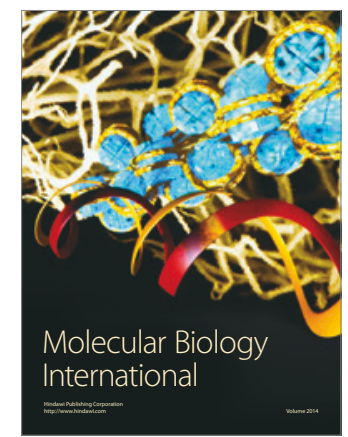

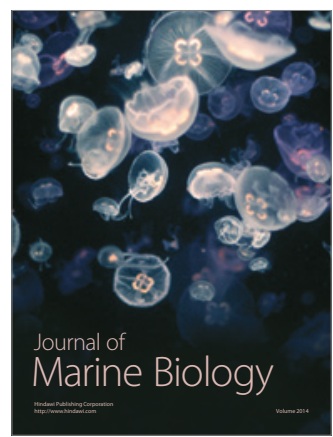

